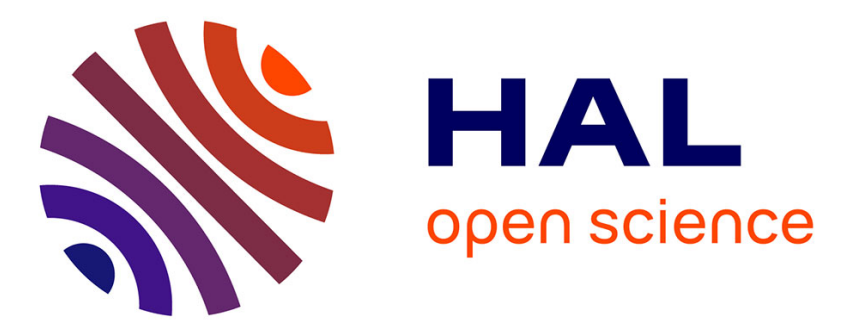

\title{
Angular acceptance analysis of an infrared focal plane array with a built-in stationary Fourier transform spectrometer
}

Frédéric Gillard, Yann Ferrec, Nicolas Guérineau, Sylvain Rommeluère, Jean Taboury, Pierre Chavel

\section{To cite this version:}

Frédéric Gillard, Yann Ferrec, Nicolas Guérineau, Sylvain Rommeluère, Jean Taboury, et al.. Angular acceptance analysis of an infrared focal plane array with a built-in stationary Fourier transform spectrometer. Journal of the Optical Society of America. A Optics, Image Science, and Vision, 2012, 29 (6), pp.936-944. 10.1364/JOSAA.29.000936 . hal-00700191

\section{HAL Id: hal-00700191 \\ https://hal-iogs.archives-ouvertes.fr/hal-00700191}

Submitted on 25 May 2012

HAL is a multi-disciplinary open access archive for the deposit and dissemination of scientific research documents, whether they are published or not. The documents may come from teaching and research institutions in France or abroad, or from public or private research centers.
L'archive ouverte pluridisciplinaire HAL, est destinée au dépôt et à la diffusion de documents scientifiques de niveau recherche, publiés ou non, émanant des établissements d'enseignement et de recherche français ou étrangers, des laboratoires publics ou privés. 


\title{
Angular acceptance analysis of an infrared focal plane array with a built-in stationary Fourier transform spectrometer
}

\author{
Frédéric Gillard, ${ }^{1}$ Yann Ferrec, ${ }^{1}$ Nicolas Guérineau, ${ }^{1, *}$ Sylvain Rommeluère, ${ }^{1}$ \\ Jean Taboury, ${ }^{2}$ and Pierre Chavel $^{2}$ \\ ${ }^{1}$ ONERA-The French Aerospace Lab, F-91761, Palaiseau, France \\ ${ }^{2}$ Laboratoire Charles Fabry, Institut d'Optique, Centre National de la Recherche Scientifique, \\ Université Paris-Sud 11, 2, Avenue Augustin Fresnel, F-91127 Palaiseau Cedex, France \\ *Corresponding author: nicolas.guerineau@onera.fr
}

Received December 21, 2011; accepted February 11, 2012; posted February 24, 2012 (Doc. ID 160351); published May 21, 2012

\begin{abstract}
Stationary Fourier transform spectrometry is an interesting concept for building reliable field or embedded spectroradiometers, especially for the mid- and far- IR. Here, a very compact configuration of a cryogenic stationary Fourier transform IR (FTIR) spectrometer is investigated, where the interferometer is directly integrated in the focal plane array (FPA). We present a theoretical analysis to explain and describe the fringe formation inside the FTIR-FPA structure when illuminated by an extended source positioned at a finite distance from the detection plane. The results are then exploited to propose a simple front lens design compatible with a handheld package. (c) 2012 Optical Society of America
\end{abstract}

OCIS codes: $\quad 040.3060,300.6190$.

\section{INTRODUCTION}

In several applications, measuring the spectral signature of objects requires handheld and robust instruments with high radiometric performance. In other words, the instrument must deliver spectra with good signal-to-noise ratios even with a low number of photons emitted by the source during a given time of acquisition. Examples include acquiring the spectrum of a chemical agent heated at a high temperature but during very small times of integration to get high rates of acquisition (up to $1 \mathrm{kHz}$ ). In other situations, samples at low temperatures (typically between 250 and $300 \mathrm{~K}$ ) have to be analyzed. In the thermal IR domain, which is the spectral domain considered in this work, special care is required to minimize the instrument's self emission and maximize the throughput of the spectrometer to increase its sensitivity.

To meet the first requirement, cooling the spectrometer [1-4] from its entrance pupil to the detection plane is the most radical solution. It ensures an optimal reduction of the background current and an excellent definition of the spectrometer geometrical étendue. In a earlier work, we have already developed an entirely cooled stationary Fourier transform spectrometer based on a Michelson interferometer in the "air wedge" configuration and an IR focal plane array (IRFPA) $[\underline{3}, 4]$. In front of the Michelson interferometer, the instrument had a front afocal lens with an intermediate image plane that imaged the scene onto an intermediate focal plane where a cold field stop was positioned. This first function ensured a good definition of the spectrometer field of view (FOV), even for scenes at low temperatures. The second function of the afocal lens was to collimate the light on the Michelson interferometer. Behind the Michelson interferometer, a rear mirror imaged the fringes localized near the Michelson mirrors onto the IRFPA. The mirrors were off-axis parabolas, and the optical chain (including mirrors and interferometer) was optimized to produce a good quality interference pattern imaged onto the IRFPA [5]. The entire optical layout was cooled down to a temperature of $77 \mathrm{~K}$ and placed in a cryostat. The instrument weighed $\sim 20 \mathrm{~kg}$, and the overall dimensions of the cryostat were $20 \mathrm{~cm}$ (in diameter) $\times 20 \mathrm{~cm}$. In order to reduce those dimensions and simplify the optical layout, we have now explored a new concept of an FTIR spectrometer $[6,7]$, which is the object of the present article. The principle is to integrate a two-wave wedge-shaped interferometer onto an IRFPA of HgCdTe technology. The device obtained is very compact $(10 \mathrm{~mm} \times 10 \mathrm{~mm})$ and can deliver spectra at high rates, corresponding to the frame rate of the FPA (up to $1 \mathrm{kHz}$ typically, depending on the readout circuit).

The second requirement (maximizing the throughput of the spectrometer) is met by choosing an optical concept able to accept as large a source as possible. In most spectroscopic problems, indeed, the instrument throughput describing the amount of useful energy detected by the spectrometer is a limiting factor. By now, as with our entirely cooled spectrometer, many existing spectrometers are based on Fourier transform (FT) spectrometry, i.e., on two-wave interferometers, like the classic Michelson setup [ㅁ-10]. The usual mode of operation is to create a time-varying delay between the two arms of the Michelson interferometer and acquire an interferogram by use of a single detector. To increase the sensitivity and operate at high rates, notice that quantum detectors cooled at liquid nitrogen temperature are used. Time-scanning FT spectrometers are widely used in a large variety of applications. This is due to the great adaptability of the concept. Indeed, the same interferometer can be used for the entire 
IR spectral range (from 1 to $30 \mu \mathrm{m}$ ), and the spectral resolution $d \sigma$ can be tuned (typically between 1 and $16 \mathrm{~cm}^{-1}$ ) by simply playing with the maximal optical path difference (OPD) $\delta_{\max }$ introduced between the two arms of the interferometer:

$$
\delta_{\max }=\frac{1}{2 d \sigma} .
$$

For applications requiring fine spectral resolutions (small $d \sigma$ ), we just have to increase the amplitude of displacement of the scanning mirror to get large optical path differences. In return, the time-scanning process leads to low rates of acquisition. In addition, the angular acceptance $\theta_{\max }$ of the spectrometer decreases with $d \sigma$. Indeed, if the criterion to define the angular acceptance $\theta_{\max }$ of a scanning FT spectrometer is that the OPD for off-axis points must not differ from the on-axis OPD by more than $\lambda / 2$ (with $\lambda$ the wavelength), then we can show that this radius $\theta_{\max }$ is given by the following relation:

$$
\theta_{\max }=\sqrt{\frac{2 d \sigma}{\sigma_{\max }}},
$$

where $\sigma_{\max }$ is the maximum detected wavenumber. It can also be shown that this angle is the maximal angular radius that ensures a fringe contrast greater than $63 \%$ for a circular source. Equation (2) shows that the angular acceptance of the spectrometer is linked to its spectral resolution. A higher resolution leads to a smaller angular acceptance. However, as seen before, our concept of compact stationary Fourier transform spectrometer (FTIR-FPA) is based on a wedge interferometer. Thus, the relation given by Eq. (2) may not be valid. That is why in this paper we focus on the angular acceptance of this device. In particular, we study the contrast of the fringes in the case of a monochromatic extended, variable size source placed at different distances from the FTIR-FPA from infinity to a close distance (of typically a few centimeters). For this, we develop a model based on the van Cittert-Zernike theorem.

In Section 2, the FTIR-FPA concept is presented with its basic performance. Two configurations are considered, depending on the manufacturing process. We show that a very high angular acceptance can be reached, compared to a classic Michelson spectrometer, in the case of an infinitely distant source. Section 3 introduces the theoretical study of fringe formation in the case of an extended source placed at a finite distance from the device. A simulation method is presented. In contrast to previous work [11], we have derived the complete formalism for both configurations. In Section $\underline{4}$, a recom- mended architecture of a miniaturized spectrometer based on our FTIR-FPA is proposed. A comparison of this kind of spectrometer with alternate designs, such as grating spectrometers, for example, is out of the scope of this article, which aims mainly at presenting the two configurations of our FTIRFPA, showing how the fringe contrast can be analytically estimated with the van Cittert-Zernike theorem, at explaining the link between this analytical calculation and the geometrical theorem on fringe localization.

\section{FTIR-FPA SPECTROMETER}

In this section, we present the concept of our FTIR-FPA spectrometer, which can be applied to two types of devices. The basic performance of each type is discussed.

\section{A. Description of the Devices}

Our FTIR-FPA concept is to miniaturize a spectrometer by merging the detector and the interferometer. Figure 1 shows schematic views of the two structures of our FTIR-FPĀ. These structures are not far from the baseline architecture of an FPA of HgCdTe diodes deposited on a CdZnTe substrate. On current generation detectors designed for backside illumination, the incident radiation first impinges the substrate, before reaching the detection layer. At the end of the classic manufacturing process, the CdZnTe wafer (typically of $500 \mu \mathrm{m}$ thickness ) is thinned down by mechanical etching to get a thin plate of a few micrometers. By use of a chemical process, this residual plate can even be completely removed. Last, in order to increase the transmission, an antireflective coating is usually deposited on the upper surface.

Our first idea (Fig. 1, left) is to modify the step of thinning in order to leave a wedge-shaped and uncoated substrate, leading to our first FTIR-FPA architecture. The consequence is that due to the abrupt changes in the refractive index at the vacuum/CdZnTe and CdZnTe/HgCdTe interfaces, internal reflections occur in the wedge, which can be considered a Fizeau interferometer, but with low reflectivity mirrors. From one given incident ray, two rays will emerge in the detection layer: the first after two refractions and the other after two refractions and two reflections inside the wedge. The other emerging rays corresponding to multiple reflections can be neglected [12]. We call this device a "monolithic" FTIR-FPA. The other idea (Fig. 1, right) is to hybridize a silicon $\left(n_{3}=3.4\right)$ prism on the surface of the sensor after having thinned the substrate completely [7]. A vacuum prism is created, leading again to two-wave interference. Note that the antireflective coating is deposited only on the upper face of the silicon wafer instead of the upper face of the active layer. We call this
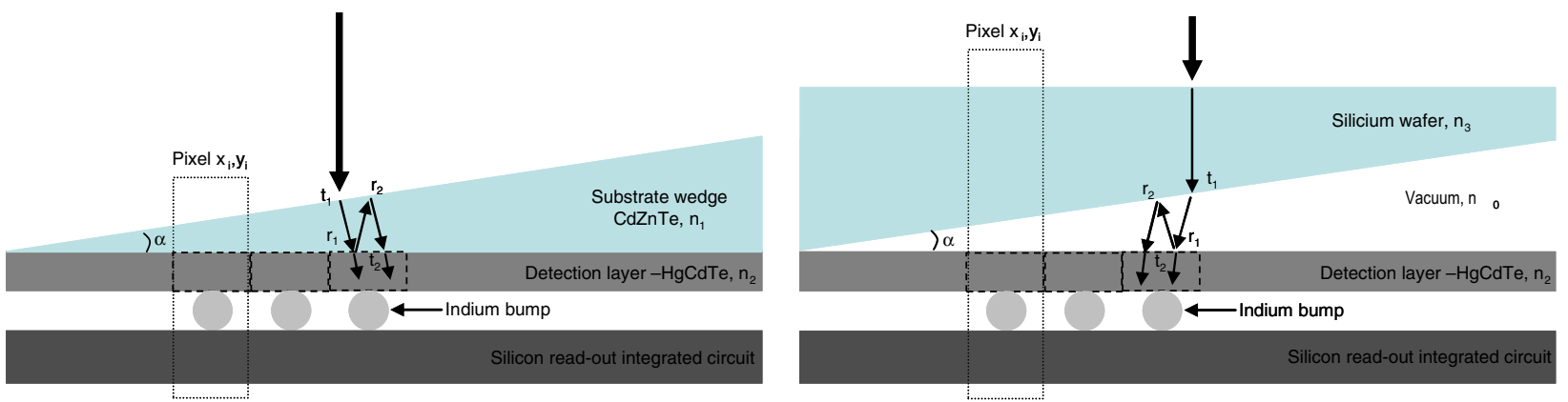

Fig. 1. (Color online) "Monolithic" FTIR-FPA concept (left) and "hybrid" FTIR-FPA concept (right). 
device a "hybrid" FTIR-FPA. For both devices, interference fringes are created inside the detection layer due to the optical path difference between the two waves.

\section{B. Basic Performance}

Each pixel $P$ of a FTIR-FPA sees an interference state that is a function of its own optical path difference $\delta_{p}$. Thus, the quantum efficiency $\eta_{P}$ of this pixel is [] $]$

$$
\eta_{P}(\sigma)=\eta_{0}+\eta_{s} \cos \left(2 \pi \sigma \delta_{P}\right)
$$

where $\sigma$ is the wavenumber, $\eta_{0}$ is the mean quantum efficiency of a pixel that includes the wedge's transmission factor $T$, and $\eta_{s}$ is the spectrometric efficiency of the structure. The FPA delivers a two-dimensional interferogram that is theoretically made of linear fringes. The optical path difference is null on one side of the FPA (edge of the prism) and maximum on the other side. This maximum optical path difference $\delta_{\max }$ sets the resolution of the device through the following equation:

$$
d \sigma=\frac{1}{2 \delta_{\max }}=\frac{1}{4 \times n_{\text {int }} \times N_{\text {pix }} \times \text { pitch } \times \tan (\alpha)} .
$$

$\alpha$ is the angle of the wedge, pitch is the size of the pixels (30 $\mu \mathrm{m}$ in our case), $N_{\text {pix }}$ is the number of pixels in the thinning direction of the FPA (320 in our case), and $n_{\text {int }}$ is the optical index of the medium that creates interference (index of the substrate for the monolithic FTIR-FPA and index of the vacuum for the hybrid FTIR-FPA). With $n_{\text {int }}=n_{0}=1$, the hybrid FTIR-FPA presents a wider spectral resolution than the monolithic one, with $n_{\text {int }}=n_{1}=2.67$ for the same size of FPA and same angle $\alpha$. For instance, a spectral resolution of $16 \mathrm{~cm}^{-1}$ is achieved with an angle $\alpha$ equal to $6 \mathrm{mrad}$ for the monolithic device, but with an angle of $16 \mathrm{mrad}$ for the hybrid device (see Table 1). Note that an increase of angle $\alpha$ leads to a narrower spectral resolution $d \sigma$ of the FTIR-FPA.

Let us now consider an extended source illuminating our FTIR-FPA (monolithic or hybrid), placed at an infinite distance from it. The spatial extension of the source will decrease the contrast of the interferogram detected. If we choose for a criterion than the fringe contrast has to be higher than 0.63 [see Eq. (17)], then we find that the maximum angular radius $\theta_{\max }$ of an incoherent circular source is given by $[\underline{12}]$

Table 1. Basic Performance of Stationary FTIR

\begin{tabular}{|c|c|c|c|}
\hline Parameters & $\begin{array}{l}\text { Monolithic } \\
\text { FTIR-FPA }\end{array}$ & $\begin{array}{c}\text { Hybrid } \\
\text { FTIR-FPA }\end{array}$ & $\begin{array}{c}\text { Classic } \\
\text { Stationary } \\
\text { FTIR } \\
\text { ("Air Wedge" } \\
\text { Michelson) }\end{array}$ \\
\hline $\begin{array}{l}\text { Spectral } \\
\text { resolution }\end{array}$ & $16 \mathrm{Cm}^{-1}$ & $16 \mathrm{Cm}^{-1}$ & $16 \mathrm{Cm}^{-1}$ \\
\hline$\alpha$ angle & $6 \mathrm{mrad}$ & $16 \mathrm{mrad}$ & $16 \mathrm{mrad}$ \\
\hline Transmission $T$ & $77 \%$ & $51 \%$ & $50 \%$ \\
\hline Fringe contrast & $14 \%$ & $57 \%$ & $100 \%$ \\
\hline $\begin{array}{l}\text { Angular acceptance } \\
\text { (infinite distance } \\
\text { source) }\end{array}$ & $34^{\circ}$ (diameter) & $13^{\circ}$ (diameter) & $13^{\circ}$ (diameter) \\
\hline
\end{tabular}
Spectrometers

$$
\theta_{\max }=\frac{n_{\mathrm{int}}}{n_{0}} \sqrt{\frac{2 d \sigma}{\sigma_{\max }}} .
$$

For the hybrid FTIR-FPA concept $\left(n_{\text {int }}=n_{0}=1\right), \theta_{\max }$ takes the form of the angular acceptance of a classic Michelson interferometer [see Eq. (2)], and the angular acceptance of the monolithic FTIR-FPA is multiplied by the factor $n_{1}$, considering the same spectral resolution. This result is true for a source placed at an infinite distance, and with the correct orientation of the FTIR-FPA with respect to the source (see Subsection 2.D).

Besides the extension of the source, two phenomena also reduce the fringe contrast. The first is the fact that due to the transmission and reflection coefficients of the diopters, the two arms of the interferometers are not well balanced. A numerical application shows that for the monolithic FTIR-FPA, the mean transmission is about $77 \%$, but with a contrast of only $14 \%$, while for the hybrid FTIR-FPA, the mean transmission is about $51 \%$ and the contrast is $57 \%$ (see Table 1). The other phenomenon that reduces the fringe contrast is the interferogram filtering due to the spatial extension of the pixel [13].

\section{Optical Architecture of the Whole Spectrometer}

In contrast with the cryogenic spectroradiometer we designed before $[3,4]$, the FTIR-FPA allows a great simplification of the optical layout, replacing by itself the Michelson interferometer and the rear lens. The next step is to simplify the front lens layout. As seen in Section 1, the front lens ensures two main functions: to define the $\overline{\mathrm{F} O V}$ by forming an image of the scene in an intermediate focal plane containing a cold stop and to collimate the light onto the FTIR-FPA. In order to get a handheld instrument compatible with the packaging of a commercial IRFPA (called an IR detector dewar cooler assembly), our idea is to suppress the collimating function of the front lens; the latter is now only necessary to define the FOV of the instrument by forming an image of the scene in the plane of the cold shield aperture. Then this image acts as an extended and incoherent secondary source placed at a finite distance that illuminates our FTIR-FPA. In that configuration, the front lens can be reduced to a classic focusing lens. Since we do not use any rear lens (the interferometer is integrated onto the FPA), the entire cryogenic instrument could be held in one hand [14]. Figure 2(b) shows this setup. To validate this minimalist approach, we conducted a theoretical study of interferogram formation in order to find the angular acceptance of our device with a source at a finite distance.

\section{Fringe Localization}

Before modeling interferograms, let us study the localization of fringes of our FTIR-FPA, first for a source at infinity and later for a source at a finite distance. The theoretical way to define the localization of fringes is the following. For each ray incident onto an interferometer, two (or more) rays will emerge. The locus of localization of fringes is defined as "the locus of the points of intersection of the rays that originate from one incident ray" [15]. At these points, the path difference variation is stationary when the source point moves. Thus an increase in the size of the source does not blur the interference fringes, at least at the first order [16,17]. 


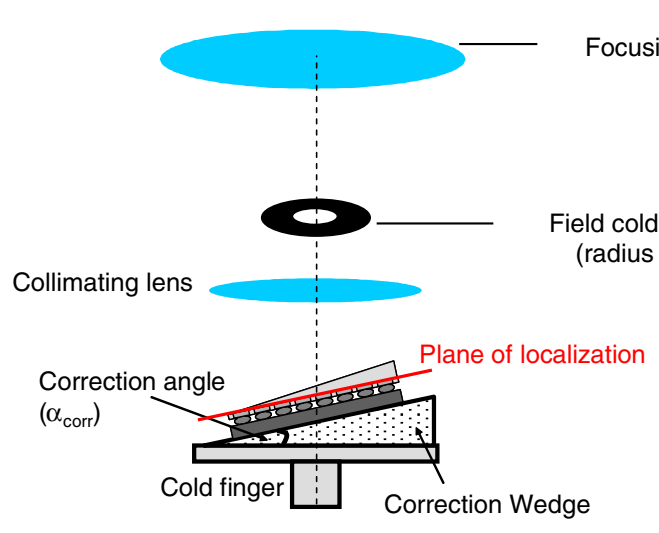

(a)

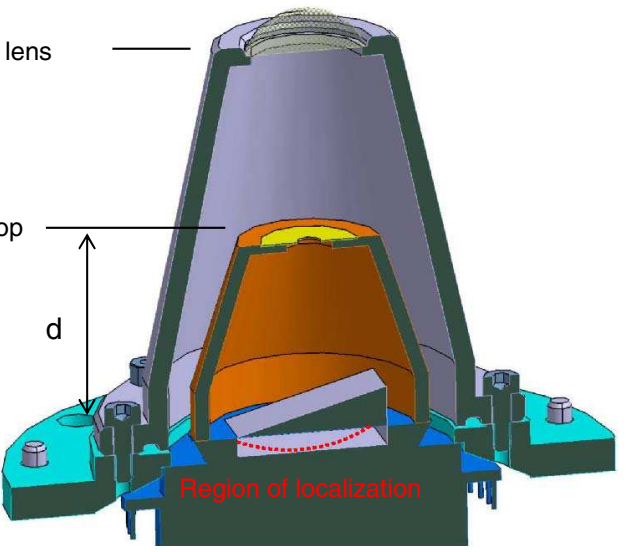

(b)

Fig. 2. (Color online) Optical layouts of a FTIR-FPA spectrometer: (a) configuration based on an afocal lens made of two lenses and the FTIR-FPA purposely tilted and (b) single-lens architecture compatible with the classic packaging of an IRFPA. As the collimating lens has been removed, the localization area is no longer a plane and thus cannot correspond to the FPA. This decreases the contrast of the detected fringes.

Let us consider our interferometer. We light our FTIRFPA with an incoherent source placed at a distance $d$. If distance $d$ is equal to infinity [see Fig. 3(a)], incident rays are parallel with an average angle $\theta$ of incidence. One of these rays passes through the prism with two refractions (first arm of the interferometer) or is reflected two times inside the index $n_{1}$ and emerges in $n_{2}$ (second arm of the interferometer). If we repeat this geometrical construction for all incident rays, it appears that the region of intersections of the two emerging rays from the same incident ray is a plane passing through the apex of the wedge. In order to optimize the fringe detection with an extended source, we should detect fringes in the region of localization. As this region is a plane, it is possible to make it coincide with the detection plane, by adding a wedge

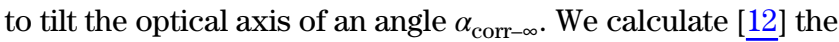
incidence of the chief ray with the formulas

$$
\alpha_{\text {corr- }-\infty}^{\text {monolithic }}=\arcsin \left[\frac{n_{1}}{n_{0}} \sin (2 \alpha)\right]-\alpha \approx \frac{2 n_{1} \alpha}{n_{0}},
$$

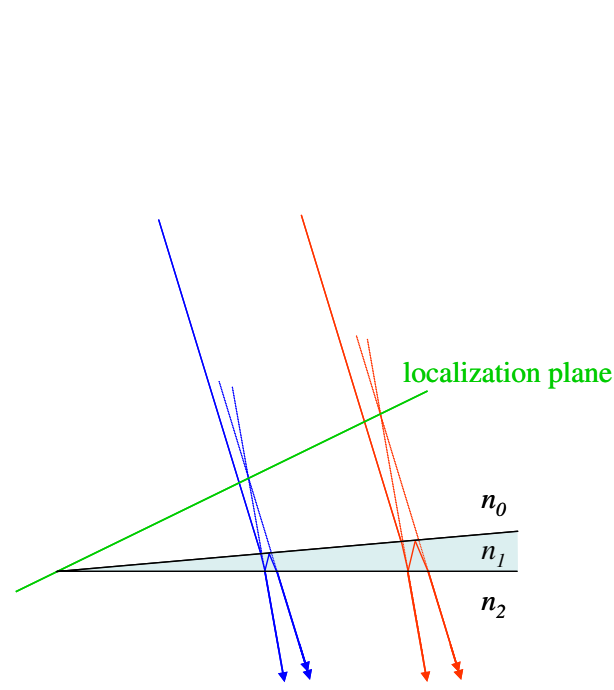

(a)

$$
\begin{aligned}
\alpha_{\text {corr- }-\infty}^{\text {hybrid }} & =\arcsin \left[\frac{n_{3}}{n_{0}} \sin \left(\arcsin \left(\frac{n_{0}}{n_{3}} \sin 2 \alpha\right)-\alpha\right)\right] \\
& \approx \alpha \cdot\left(2-\frac{n_{3}}{n_{0}}\right) .
\end{aligned}
$$

It means that after the reflection at the bottom of the cavity, the chief ray is in autocollimation with respect to the upper mirror of the cavity (see Fig. 4). With $\alpha=6 \mathrm{mrad}$, $n_{1}=2.67$, and $n_{0}=1$, the correction angle for a source at infinity $\alpha_{\text {corr- } \infty}$ is equal to $1.8^{\circ}$ for the monolithic FTIR-FPA. With $\alpha=16 \mathrm{mrad}, n_{3}=3.4$, and $n_{0}=1$, this correction angle becomes equal to $-1.3^{\circ}$ for the hybrid FTIR-FPA. On a practical stage, the tilt of the detection plane avoids tilting the optical axis of the rear lens [see Fig. 2(a)].

If $d$ now has a finite value [see Fig. 3(b)], which is the case of interest, the same construction to determine the region of localization leads to the construction of two virtual sources, $S_{1}$ and $S_{2}$, images of the real source $S$ through the two arms of

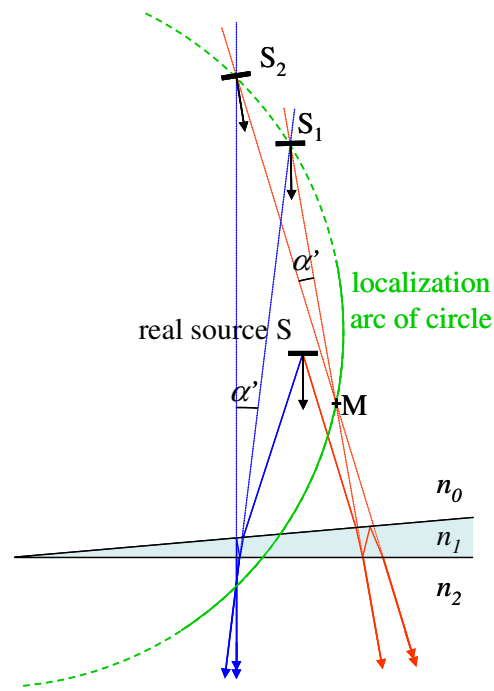

(b)

Fig. 3. (Color online) Surfaces of localization of fringes: (a) with an infinite distance to the source, (b) with a finite distance $d$ to the source. In the latter case, and due to refractive effects, the area of localization approximates a circle only locally. 

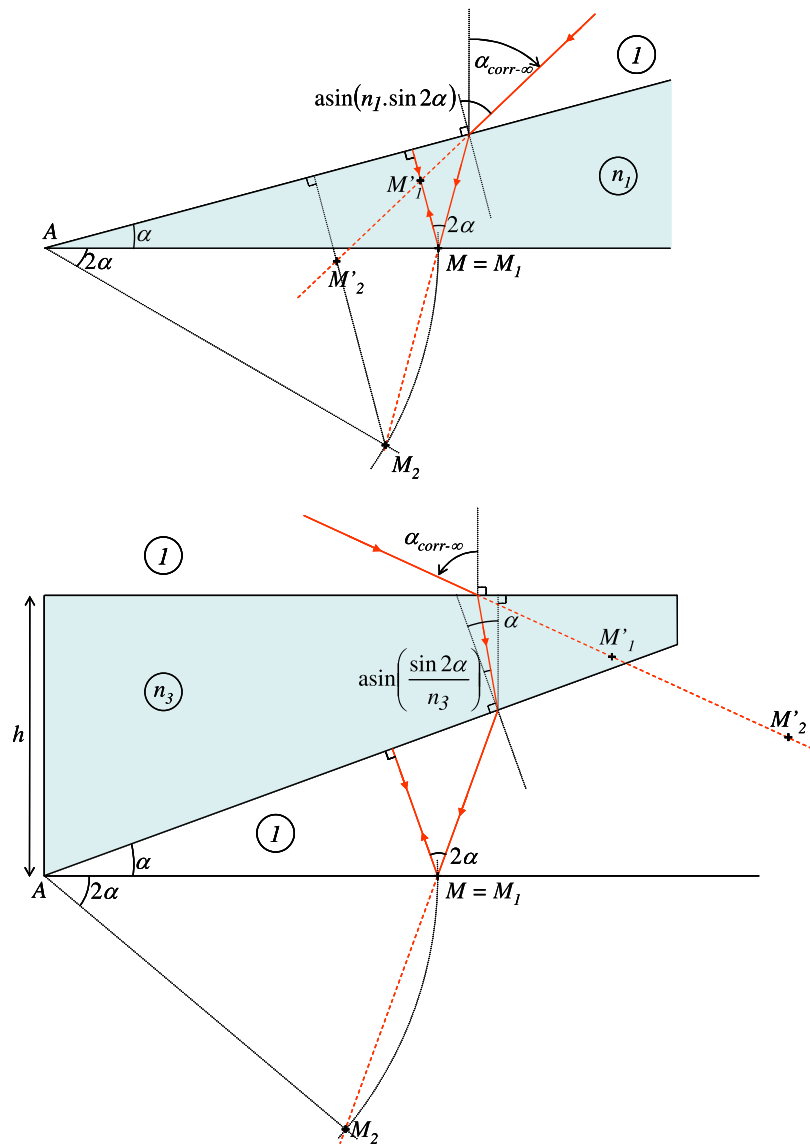

Fig. 4. (Color online) Geometrical construction of the rays to obtain fringes localized in the detection plane, for the monolithic FTIR-FPA (top) and for the hybrid FTIR-FPA (bottom). $M_{1}^{\prime}$ and $M_{2}^{\prime}$ are the images of $M_{1}$ and $M_{2}$ in the source space. When the $\left(M_{1}^{\prime} M_{2}^{\prime}\right)$ line goes through the center of the source, then $M$ belongs to the region of localization.

the interferometer. We call the angle formed by the two rays emerging from one incident ray $\alpha^{\prime}$. Its value depends on $\alpha, n_{1}$, and $n_{2}$, but in the paraxial approximation, this angle is constant for all incident rays: it means that if point $M$ belongs to the area of localization, then angle $S_{1} M S_{2}$ is equal to $\alpha^{\prime}$, which is independent from $M$ in the paraxial approximation. As can be shown by geometrical arguments (inscribed angle theorem), the locus of point $M$ verifying that angle $S_{1} M S_{2}$ is constant is a circle. Thus, in the plane that contains the center $S_{0}$ of the source $S$ and is orthogonal with the prism edge, the region of localization of fringes is a circle passing through the center of the two virtual sources. In the other planes, there is no intersection between those two emerging rays, except the apex of the cavity, obviously. The same geometrical construction leads to the same conclusion for the hybrid FTIR-FPA.

Note that the assertion of the angle formed by the two rays emerging from one incident ray is only valid in the paraxial approximation. Therefore, the conclusion that the localization area is the circle is only locally valid, unless there is no refraction inside the wedge, i.e., if $n_{0}=n_{1}=n_{2}$. This is, for instance, the case with a wedge Michelson interferometer [18;19, pp. 291-300]: images $S_{1}$ and $S_{2}$ of $S$ are perfectly stigmatic, and the whole localization area is a circle, passing through $S_{1}, S_{2}$, and the apex of the wedge.

Thus, when the source is imaged at a finite distance from the FTIR-FPA, the locus of fringe localization is not a plane, and hence, it is not possible to make it coincide with the detection plane. The angular acceptance of the FTIR-FPA is consequently liable to be significantly reduced compared with the expression given by Eq. (5). A more elaborate study is needed to quantify the fringe contrast loss. This study is the subject of the next section.

\section{INTERFEROGRAM MODELING}

In this section, we shall present the technique used to model FTIR-FPA interferograms in the case of a finite distance illumination.

\section{A. Interferogram Equation}

The geometry of the problem is presented in Fig. 5. This geometry is the monolithic FTIR-FPA one. Let us consider a quasimonochromatic (wavelength $\lambda$ ) extended, incoherent, and plane source $S$ placed in the air at a distance $d$ from the $n_{1} / n_{2}$ interface. For a given incident ray, a first ray emerges after two refractions at the interfaces $n_{0} / n_{1}$ and $n_{1} / n_{2}$; this path corresponds to the first arm of the interferometer. A second ray emerges after two reflections inside the wedge; this path corresponds to the second arm of the interferometer. In this paper, we shall always consider that all of the energy that reaches the active layer is instantly absorbed, and thus we call the upper side of the active layer the "detection plane."

As illustrated in Fig. 5, we can consider $M_{1}^{\prime}$ and $M_{2}^{\prime}$ as the images (supposed to be stigmatic) in the source space of a point $M$ in the detection layer of index $n_{2}$ through the two arms of the interferometer. When this structure is illuminated by a source $S$, interferences at point $M$ are the interferences between $M_{1}^{\prime}$ and $M_{2}^{\prime}$ [20]. Contrast of fringes in $M$ depends on the degree of mutual coherence between $M_{1}^{\prime}$ and $M_{2}^{\prime}$. More precisely, the intensity $I(M)$ at point $M$ is given by $[\underline{19}$, pp. 499-503]

$$
I(M)=I\left(M_{1}^{\prime}\right)+I\left(M_{2}^{\prime}\right)+2 \sqrt{I\left(M_{1}^{\prime}\right) I\left(M_{2}^{\prime}\right)} \cdot\left|\gamma_{12}\right| \cdot \cos \left(\alpha_{12}\right) .
$$

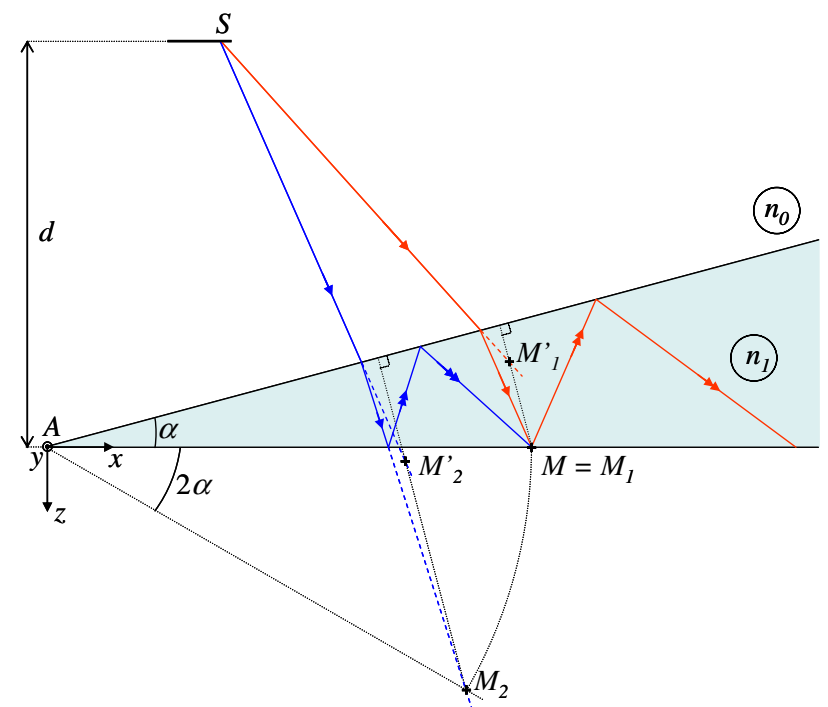

Fig. 5. (Color online) Geometry of the problem for a monolithic structure. Note that it is not the same incident ray that reaches $M$ through the two arms of the interferometer (red ray for the first arm, blue ray for the second arm); $M$ does not belong to the localization circle. 
In this equation, $\left|\gamma_{12}\right|$ and $\alpha_{12}$ are the modulus and the phase of the complex degree of coherence $\gamma_{12}$ of the light.

We want to study the contrast $C(M)$ of the interference phenomena at point $M$. According to Eq. ()ㅡ, $C(M)$ is given by

$$
C(M)=\frac{2 \sqrt{I\left(M_{1}^{\prime}\right) I\left(M_{2}^{\prime}\right)}\left|\gamma_{12}\right|}{I\left(M_{1}^{\prime}\right)+I\left(M_{2}^{\prime}\right)} .
$$

We call the amplitude reflection coefficients of the upper mirror of the cavity and of the bottom mirror $r_{1}$ and $r_{2}$. We can then write

$$
C(M)=\frac{2 r_{1} r_{2}}{1+\left(r_{1} r_{2}\right)^{2}} \cdot\left|\gamma_{12}(M)\right|
$$

If source $S$ were perfectly spatially coherent, $\left|\gamma_{12}\right|$ would be strictly equal to 1 , and the contrast of fringes would be equal to the ratio expressed in Eq. (10). A numerical application (considering that incident angles are small) gives us a value of $14 \%$ for the monolithic FTIR-FPA and 57\% for the hybrid FTIR-FPA (these values were presented in Table 1[21]). As source $S$ is not perfectly coherent, we must calculate the complex degree of coherence at point $M$. In order to do that, we use the van Cittert-Zernike theorem that gives the value of the degree of coherence at point $M$. This value is a function of the $M_{1}^{\prime}$ and $M_{2}^{\prime}$ positions. In the two next subsections, we shall explain how we determine these positions. Then we shall expose the principle of the contrast estimation.

\section{B. Location of Points $M_{1}^{\prime}$ and $M_{2}^{\prime}$}

The expression of the location of points $M_{1}^{\prime}$ and $M_{2}^{\prime}$ is easily written thanks to the stigmatism hypothesis between an object and its image through a plane surface. Indeed, if the object is located at distance $h$ from the surface, then its image remains on the normal to the surface that goes through the object point, but at a distance $h \cdot n_{\text {img }} / n_{\text {obj }}$ from the surface, with $n_{\text {obj }}$ the refractive index of the object space and $n_{\text {img }}$ the refractive index of the image space. Thus, the operation to go from the detection space to the source space is a linear transformation and can be written as a matrix $M_{\text {det } \rightarrow \text { source }}$, the product of elementary translation, rotation, and dilatation matrixes. Moreover, we only consider points $M$ lying at the top of the active layer. Consequently, the refraction that occurs when entering the active layer can be given up. For the monolithic FTIR-FPA, the $M_{\text {det } \rightarrow \text { source }}$ matrix is given by
The conjugate of $M_{1}^{\prime}$ in the detection space is $M_{1}$, equal to $M$, and the conjugate of $M_{2}^{\prime}$ is $M_{2}$, deduced from $M$ by a rotation of angle $2 \alpha$ around the apex of the wedge ( $y$ axis). Point $M$ is defined by its affine coordinates $(x, y, 0,1)(z=0$ since we limit $M$ to be at the upper surface of the active layer): the referential, both in the detection and the source space, is $(A, x, y, z)$ as defined on Fig. 4 . Additional translation and rotation matrixes will be used to properly position the source with respect to the FPA.

\section{Computation of the Complex Degree of Coherence}

According to Eq. (10), the computation of the complex degree of coherence is the key of the interferogram modeling. We must know the value of $\gamma_{12}$ at point $M$. For this calculation, we use the van Cittert-Zernike theorem between points $M_{1}^{\prime}$ and $M_{2}^{\prime}$, images of $M$ in the source space through the two arms of the interferometer. We consider that the source $S$ is extended, plane, incoherent, monochromatic (of wavelength $\lambda$ ), and uniform. Its luminance is $L(\vec{s})$. In the paraxial approximation, expanding the mutual coherence formula between disturbances in $M_{1}^{\prime}$ and $M_{2}^{\prime}$ leads to Eq. (13), below. Vectors $\vec{r}_{M_{1}}$ and $\vec{r}_{M_{2}}$ are the position vectors of $M_{1}^{\prime}$ and $M_{2}^{\prime}$ in a plane parallel to the source. $d_{1}$ and $d_{2}$ are the distances along the $z$ axis between the plane of the source and points $M_{1}^{\prime}$ and $M_{2}^{\prime}$, respectively. If $d_{1}=d_{2}$, we recover the classical van Cittert-Zernike formula, where $\gamma_{12}(M)$ is directly related to the Fourier transform of the source spatial luminance $[\underline{19}$, p 510]:

$$
\begin{aligned}
& \gamma_{12}(M)=\exp \left(i k\left(d_{1}-d_{2}\right)\right) \cdot \exp \left(\frac{i \pi}{\lambda}\left(\frac{\vec{r}_{M_{1}}^{2}}{d_{1}}-\frac{\vec{r}_{M_{2}}^{2}}{d_{2}}\right)\right) \\
& \times \frac{\iint L(\vec{s}) \cdot \exp \left(\frac{i \pi}{\lambda}\left(\frac{1}{d_{1}}-\frac{1}{d_{2}}\right) s^{2}\right) \cdot \exp \left(\frac{2 i \pi}{\lambda}\left(\frac{\vec{r}_{M_{2}}}{d_{2}}-\frac{\vec{r}_{M_{1}}}{d_{1}}\right) \vec{s}\right) d^{2} s}{\tilde{L}(0)} .
\end{aligned}
$$

$M$ belongs to the region of localization for

$$
\frac{\vec{r}_{M_{2}}}{d_{2}}-\frac{\vec{r}_{M_{1}}}{d_{1}}=0 .
$$

It means that the optical path difference $S M_{1}^{\prime}-S M_{2}^{\prime}$ does not vary at the first order when $S$ moves. The remaining quadratic term in the integral is responsible of the limitation of the angular acceptance of this wedge interferometer even when we

$$
M_{\text {det } \rightarrow \text { source }}^{\text {monolithic }}=\left(\begin{array}{cccc}
\cos ^{2} \alpha+\sin ^{2} \alpha / n_{1} & 0 & -\sin \alpha \cos \alpha \cdot\left(1-1 / n_{1}\right) & 0 \\
0 & 1 & 0 & 0 \\
-\sin \alpha \cos \alpha \cdot\left(1-1 / n_{1}\right) & 0 & \sin ^{2} \alpha+\cos ^{2} \alpha / n_{1} & 0 \\
0 & 0 & 0 & 1
\end{array}\right)
$$

For the hybrid FTIR-FPA, this matrix becomes

$$
M_{\text {det } \rightarrow \text { source }}^{\text {hybid }}=\left(\begin{array}{cccc}
\cos ^{2} \alpha+n_{3} \sin ^{2} \alpha & 0 & \sin \alpha \cos \alpha \cdot\left(n_{3}-1\right) & 0 \\
0 & 1 & 0 & 0 \\
\sin \alpha \cos \alpha \cdot\left(1-1 / n_{3}\right) & 0 & \cos ^{2} \alpha+\sin ^{2} \alpha / n_{3} & -h \cdot\left(1-1 / n_{3}\right) \\
0 & 0 & 0 & 1
\end{array}\right) .
$$


detect fringes in the localization area [16]. Indeed, we then have

$$
\left|\gamma_{12}(M)\right|=\frac{\iint L(\vec{s}) \cdot \exp \left(\frac{i \pi s^{2}}{\lambda d_{\mathrm{eq}}}\right) d^{2} s}{\int L(\vec{s}) d^{2} s},
$$

with

$$
\frac{1}{d_{\mathrm{eq}}}=\frac{1}{d_{1}}-\frac{1}{d_{2}} \approx \frac{\delta}{n_{\mathrm{int}}^{2} \cdot d^{2}},
$$

$\delta$ being the optical path difference at point $M$. Thus, for a circular source of radius $r_{0}$ located at distance $d$, we obtain

$$
\left|\gamma_{12}(M)\right|=\operatorname{sinc}\left(\frac{\mathrm{r}_{0}^{2}}{2 \lambda d_{\mathrm{eq}}}\right) \approx \operatorname{sinc}\left(\frac{\mathrm{r}_{0}^{2} \delta}{2 \lambda d^{2} n_{\text {int }}^{2}}\right)
$$

if fringes are localized at $M$. As stated above, this is true if $\vec{r}_{M_{1}} / d_{1}=\vec{r}_{M_{2}} / d_{2}$. It means that the two images $M_{1}^{\prime}$ and $M_{2}^{\prime}$ of $M$ in the entrance space are aligned with the source center (see Fig. $\underline{6}$, top).

Besides, we know that the line $\left(M_{1}^{\prime} M_{2}^{\prime}\right)$ makes an angle

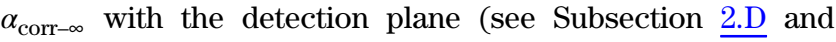
Fig. 4). Therefore, by tilting the FTIR-FPA around the $y$ axis (angle $\alpha_{\text {corr- } M}$ on Fig. 6 , bottom), we can choose the position of the intersection of the localization circle with the detection plane, while maintaining the FTIR-FPA centered on the source axis. At this point, the modulus of the degree of coherence, deduced without any approximation from Eq. (13), is given by Eq. (17).

When $\bar{M}$ is not within the localization area, then the optical path difference between $S M_{1}^{\prime}$ and $S M_{2}^{\prime}$ varies with $S$ at the first order. Thus, the linear term in the integral of Eq. (13) will be the first responsible for the drop of the fringe contrast, before
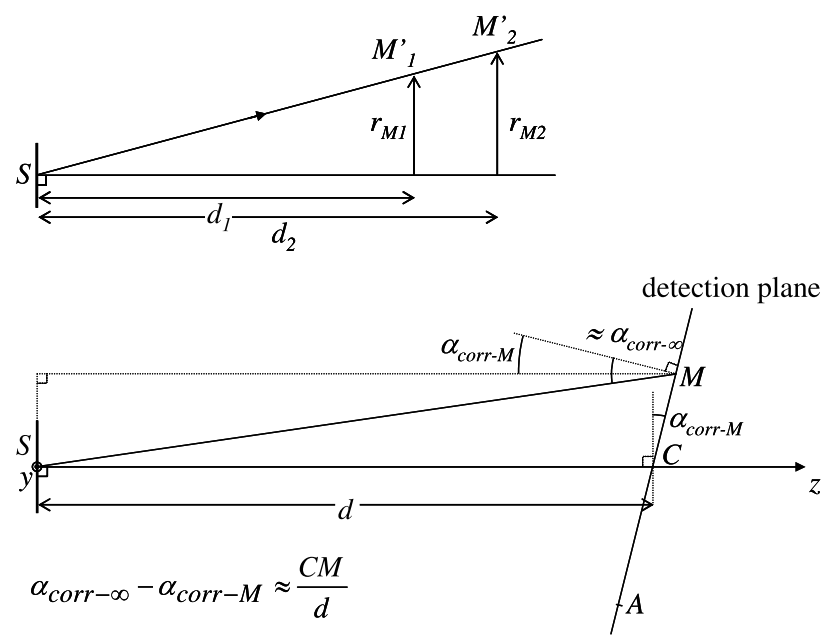

Fig. 6. Top: Fringes are localized in $M$ if its images $M_{1}^{\prime}$ and $M_{2}^{\prime}$ in the source space through the two arms of the interferometer (not shown on this figure) are aligned with the source center: $\vec{r}_{M_{1}} / d_{1}=\vec{r}_{M_{2}} / d_{2}$. Bottom: An appropriate orientation of the detection plane with respect to the source allows control of where this plane intersects the localization circle (at point $M$ in this case), while the source aligned with the center $C$ of the FTIR-FPA is maintained. Note that this drawing is only an approximation, since, strictly speaking, $M$ does not belong to line $\left(M_{1}^{\prime} M_{2}^{\prime}\right)$. the quadratic term, which can then be neglected. More precisely, if

$$
\mathrm{r}_{0}^{2}<\lambda d_{\mathrm{eq}}
$$

then this quadratic term is lower than $\pi$. For the monolithic FTIR-FPA, we have $d_{\text {eq }} \approx d^{2} \cdot n_{1}^{2} / \delta$, with $d_{\text {eq }} \approx d^{2} / \delta$ for the hybrid FTIR-FPA; not surprisingly, we find that the angular size of the source has to be lower than the criterion given by Eq. (5). Then, the modulus of the degree of coherence is reduced to the normalized Fourier transform of the spatial source luminance $L(\vec{s})$ at point $\vec{r}_{M_{2}} / d_{2}-\vec{r}_{M_{1}} / d_{1}$. If the source is circular with a radius of $r_{0}$, we have

$$
\left|\gamma_{12}\right|=\left|\frac{2 J_{1}\left[2 \pi \frac{r_{0}}{\lambda}\left|\frac{\vec{r}_{M_{2}}}{d_{2}}-\frac{\vec{r}_{M_{1}}}{d_{1}}\right|\right]}{2 \pi \frac{r_{0}}{\lambda}\left(\frac{\vec{r}_{M_{2}}}{d_{2}}-\frac{\vec{r}_{M_{1}}}{d_{1}}\right)}\right|,
$$

where $J_{1}$ is the Bessel function of the first order.

\section{NUMERICAL RESULTS}

We consider both the monolithic and the hybrid FTIR-FPAs described in Section 1. These two spectrometers have a similar FPA consisting of $320 \times 256$ pixels with a pitch of $30 \mu \mathrm{m}$. For the monolithic FTIR-FPA, the CdZnTe substrate $\left(n_{1}=2.67\right)$ is thinned with an angle of $6 \mathrm{mrad}$. For the hybrid FTIR-FPA, the cavity is created by a silicon wedge $(n=3.4)$ with an angle equal to $16 \mathrm{mrad}$. Thus, we have the same maximum optical path difference (about $300 \mu \mathrm{m}$ ) for both spectrometers. We consider the source $S$ to be circular, of radius $r_{0}$, monochromatic $(\lambda=3 \mu \mathrm{m})$, plane, incoherent, and spatially uniform. We place it at a distance $d$ from the detector, centered on the middle of the detector. The FTIR-FPAs are tilted, so that the intersection of the localization circle and the plane detection occurs at row number 18 (middle of the FPA) and at column 280 (the apex of the wedge is at column 0 ). The modulus of the degree of coherence is estimated according to Eq. (19).

Figure $\underline{7}$ shows the computed contrast $C$ versus the coordinates $(\bar{x}, y)$ in the detection plane for the monolithic FTIRFPA. The radius of the source is $1 \mathrm{~mm}$, and its distance from

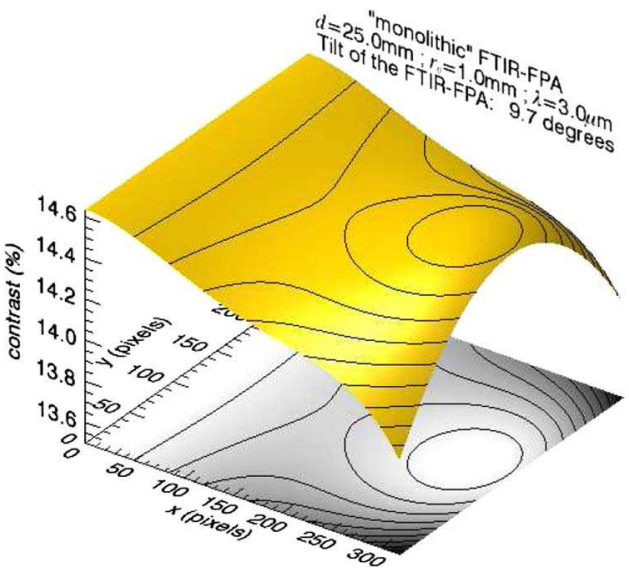

Fig. 7. (Color online) Monolithic FTIR-FPA contrast in the detection plane for $d=25 \mathrm{~mm}$ and $r_{0}=1 \mathrm{~mm}(\lambda=3 \mu \mathrm{m})$. 


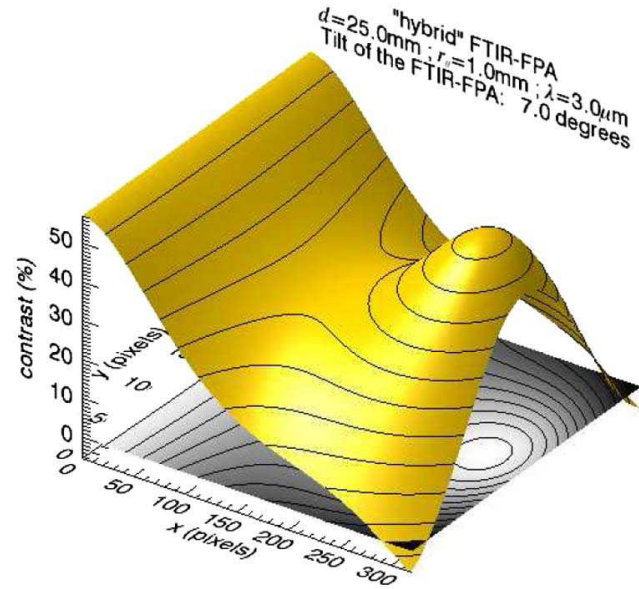

Fig. 8. (Color online) Hybrid FTIR-FPA contrast in the detection plane for $d=25 \mathrm{~mm}$ and $r_{0}=1 \mathrm{~mm}(\lambda=3 \mu \mathrm{m})$.

the detection plane is $25 \mathrm{~mm}$ (angular diameter of $4.6^{\circ}$ ). We can see a decrease of the contrast with increase in $x$. The contrast is maximum on the apex of the prism $(x=0)$. This contrast would be $100 \%$ if the two arms of the interferometer were equilibrated. With the equi-contrast lines plotted on the surface and with the projected image, we show that in the plane of the source, the degree of coherence decreases before reaching a local maximum. This is the mark of the intersection of the detection plane with the localization circle, which is well at column 280. Notice that the drop in contrast is very slight here. With a decrease of $d$ or an increase of $r_{0}$, the drop in contrast would be more important. Figure 8 shows the same computation on the hybrid FTIR-FPA. Again, the mark of the maximum circle of contrast is present. As expected, the contrast drop is greater than in the monolithic case; there is even a contrast inversion in the corners of the FPA opposite to the apex.

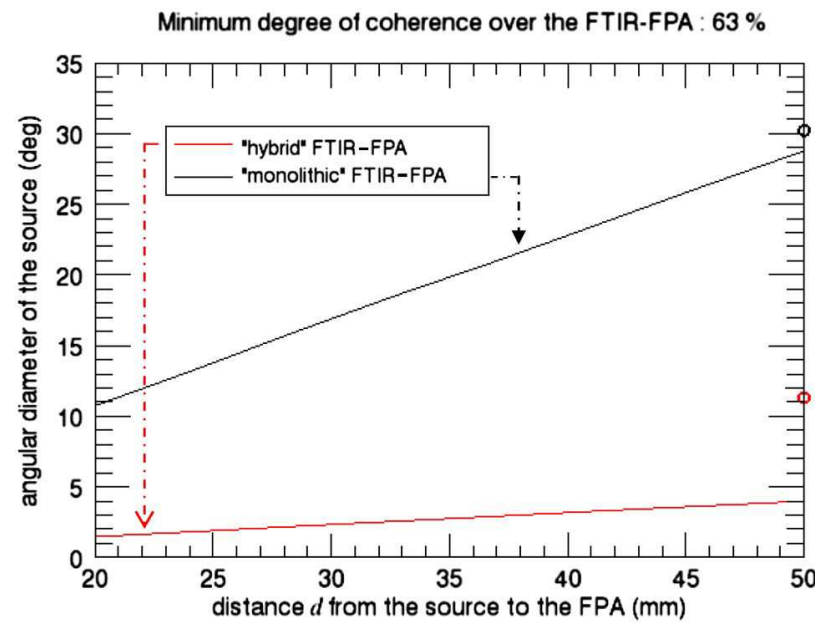

Fig. 9. (Color online) Angular acceptance of the FTIR-FPAs as a function of the distance from the source $(\lambda=3 \mu \mathrm{m})$. The degree of coherence is $63 \%$ over the whole FPA. The circles represent the angular diameter of a source at infinity, which would give the same minimum degree of coherence (see Eq. 5). Note that theses circles are calculated with Eq. (13), while we neglected the quadratic term of this integral to calculate the solid curves; on these latter, the effect of the approximation may become noticeable when the diameter of the source is close to the one indicated by the circles.
Next, we consider the maximum angular size of the source as a function of its distance to the detector for a given contrast threshold. This criterion is the minimum of contrast in the detection plane. The results are plotted in Fig. 9. This figure again emphasizes the greater angular acceptance of the monolithic FTIR-FPA compared with the hybrid FTIR-FPA, but also the fact that with an incoherent source at a finite distance, it is not possible to detect fringes in their localization area (at least with a planar FPA).

\section{DISCUSSION}

As expected, the fact that fringes are detected outside their area of localization limits the acceptable size of the source, and therefore the entrance flux. This effect is very heavy for the hybrid FTIR-FPA compared with the monolithic device: if $d=25 \mathrm{~mm}$, the angular diameter of the source is limited to $13.8^{\circ}\left(r_{0}=3 \mathrm{~mm}\right)$, while it is only $1.9^{\circ}\left(r_{0}=0.4 \mathrm{~mm}\right)$ for the hybrid FTIR-FPA. The geometrical étendue of the latter is 50 times lower than that of the monolithic FTIR-FPA $\left(6.5 \times 10^{-8} \mathrm{~m}^{2} \cdot \mathrm{sr}\right.$ versus $\left.3.3 \times 10^{-6} \mathrm{~m}^{2} \cdot \mathrm{sr}\right)$. This loss is far more significant than the advantage of the hybrid device regarding the product transmission $\times$ contrast $(30 \%$ versus $10 \%)$. This means that the hybrid concept may not be appropriate for this very compact solution with a source at a finite distance (as stated at the beginning of this article, a second lens placed after the cold shield aperture would allow a substantial increase in the angular acceptance, but at the expense of the compactness of the system).

However, one must note (see Fig. 10) that the average contrast over the FTIR-FPA is notably greater than the minimum contrast, which was our criterion; an increase in the size of the source rapidly leads to a very poor contrast in the corners of the FPA opposite to the wedge apex. Nevertheless, the contrast may remain acceptable on a wide area of the detector. Furthermore, some applications involve very luminous sources (for instance, on a combustion test bench), and in other cases it is possible to average a huge number of acquisitions. In these cases, temporal noise is reduced, and the limiting factor becomes the defects of the nonuniformity correction. As this noise is more or less a multiplicative noise, the hybrid component is more suitable than the monolithic

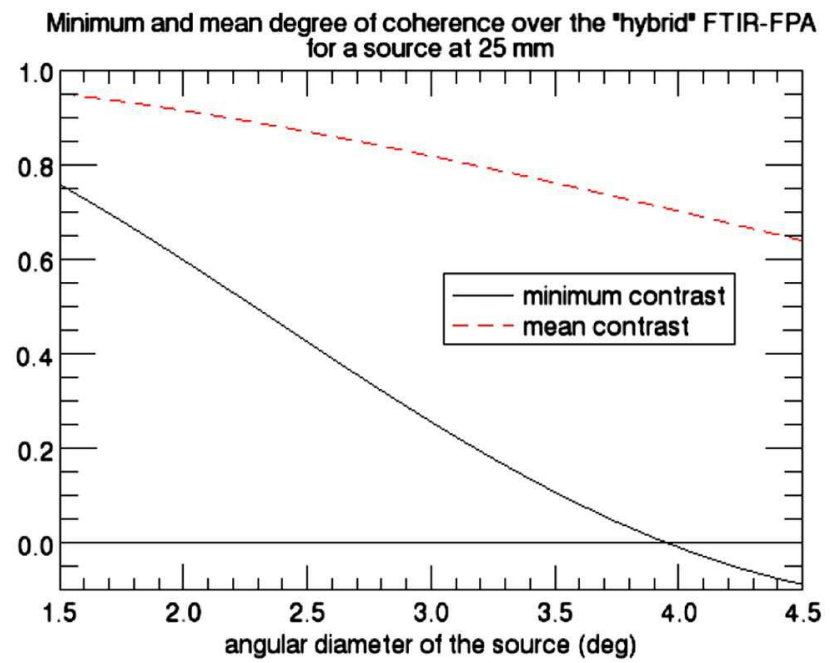

Fig. 10. (Color online) Minimum and mean degree of coherence for the hybrid FTIR-FPA 
one. Besides, other criteria have to be considered: access to the zero path difference (an advantage of the hybrid device, because in this area the photodiodes may be affected by the thinning process for the monolithic FTIR-FPA), sturdiness in front of a vibrating environment like a combustion test bench (an advantage of the monolithic device), and impact of the parasitic reflections inside the wedge. Nonetheless, its wide angular acceptance makes the monolithic device a preferential solution for ultracompact cryogenic IR spectrometers.

\section{CONCLUSION}

We presented in this paper a theoretical and numerical analysis of a stationary Fourier transform spectrometer design, integrated in the structure of an IRFPA. The theoretical performance in terms of angular acceptance was derived for two structures, called "monolithic" and "hybrid." The monolithic configuration involves a wedge-shaped substrate that exhibits a high angular acceptance thanks to the substrate's high index of refraction. The hybrid structure includes an air wedge that exhibits a lower angular acceptance but produces optical fringes with better contrast. A simplified design without a rear lens compatible with a handheld cryogenic instrument was proposed. In order to estimate its performance, we modeled the fringe formation inside the structure using the van CittertZernike theorem. We saw that our calculation retrieves the localization regions predicted by the theory. More precisely, in the case of a source placed at a finite distance from the detection plane, the computed curves of fringe contrast exhibit a local maximum where the circle of localization intersects with the detection plane. Finally, we demonstrated that with an extended source placed at a finite distance from the detector, our monolithic device keeps its high angular acceptance. This result paves the way to the design and realization of highétendue, cryogenic IR spectrometers embedded in a handheld package.

\section{REFERENCES AND NOTES}

1. J. Giroux, D. Lamarre, J. McKinnon, and H. L. Buijs, "Commercial cryogenic Fourier transform spectrometer for emission measurements of materials," Proc. SPIE 1575, 205-206 (1992).

2. S. G. Kaplan, S. I. Woods, T. M. Jung, and A. C. Carter, "Cryogenic Fourier transform infrared spectrometer from 4 to 20 micrometers," Proc. SPIE 7739, 77394D (2010).

3. Y. Ferrec, S. Rommeluère, D. Henry, and N. Guérineau, "First results from Mistere, a cryogenic static Fourier-transform spectroradiometer," in Fourier Transform Spectroscopy, OSA Technical Digest (CD) (Optical Society of America, 2009), paper FMB6.

4. N. Guérineau, S. Suffis, P. Cymbalista, and J. Primot, "Conception of a stationary Fourier transform infrared spectroradiometer for field measurements of radiance and emissivity," Proc. SPIE 5249, 441-448 (2004).
5. H. Sauer, Y. Ferrec, C. Armellin, J. Taboury, P. Cymbalista, N. Guérineau, M-A Martin, and J. Primot, "Accurate modeling of optical system aberrations applied to the design of a stationary Fourier transform spectroradiometer," Proc. SPIE 5962, 596212 (2005).

6. S. Rommeluère, N. Guérineau, R. Haidar, J. Deschamps, E. De Borniol, A. Million, J-P Chamonal, and G. Destefanis, "Infrared focal plane array with a built-in stationary Fourier-transform spectrometer: basic concepts," Opt. Lett. 33, 1062-1064 (2008).

7. M. Fendler, G. Lasfargues, S. Bernabé, G. Druart, F. de la Barrière, S. Rommeluère, N. Guérineau, N. Lhermet, and H. Ribot, "Integration of advanced optical functions on the focal plane array for very compact MCT-based micro cameras," Proc. SPIE 7660, 766022 (2010).

8. R. J. Bell, Introductory Fourier Transform Spectroscopy (Academic, 1972), pp. 1-15.

9. A. R. Korb, "Portable Fourier transform infrared spectroradiometer for field measurements of radiance and emissivity," Appl. Opt. 35, 1679-1692 (1996).

10. W. Noell, P. A. Clerc, L. Dellmann, B. Guldimann, H. P. Herzig, O. Manzardo, C. Marxer, K. Weible, R. Dändliker, and N. De Rooij, “Applications of SOI-based optical MEMS," IEEE J. Sel. Top. Quantum Electron. 8, 148-154 (2002).

11. F. Gillard, N. Guérineau, S. Rommeluère, J. Taboury, and P. Chavel, "Fundamental performances of a micro stationary Fourier transform spectrometer," Proc. SPIE 7716, 77162E (2010).

12. S. Rommeluère, "Intégration d'un micro-spectromètre statique par transformée de Fourier sur un plan focal infrarouge," Ph.D. thesis (Université Paris Sud, 2007).

13. P. Voge and J. Primot, "Simple infrared Fourier transform spectrometer adapted to low light level and high-speed operation," Opt. Eng. 37, 2459-2466 (1998).

14. N. Guérineau, G. Druart, F. de la Barrière, F. Gillard, S. Rommeluère, J. Primot, J. Deschamps, J. Taboury, and M Fendler, "Micro-camera and micro-spectrometer designs adapted to large infrared focal plane arrays," Proc. SPIE 7716, $77160 \mathrm{~N}$ (2010).

15. M. Françon, "Localization and visibility of fringes," in Optical Interferometry (Academic, 1966), pp. 62-67.

16. G. Fortunato and P. Jacquinot, "Recherche de l'étendue maximale dans les interféromètres,” C. R. Acad. Sci. Paris 274-B, 688-691 (1972).

17. J. C. Wyant, "Fringe localization," Appl. Opt. 17, 1853 (1978).

18. P. Hariharan, Optical Interferometry (Academic, 1986), p. 23.

19. M. Born and E. Wolf, Principles of Optics, 6th ed. (Pergamon, 1980).

20. In fact, one should also take into account the optical path to go from $M$ to its image $M_{1}$ by the first arm of the interferometer, and the one to go from $M$ to its image $M_{2}$ by the second arm of the interferometer. Indeed, the stigmatism hypothesis between $M$ and $M_{1}$, for instance, means only that the path to go from $M$ to $M_{1}$ does not depend on the considered ray, but it does depend on point $M$ or on the considered arm. However, this is only a phase term, and as long as we are interested mainly in the contrast of the fringes, we will omit this term.

21. Considering large incident angles, we should use the Fresnel formula for each polarization. Nevertheless, in the rest of this article, we will only use the Fresnel coefficients under normal incidence. 